\title{
Arte y Educación: Espacios comunes. Reflexiones en torno a la práctica artística Mapa dels desitjos
}

\author{
Art and Education: Common Places. Reflections surrounding artistic \\ practice in Mapa dels desitjos (Map of Wishes)
}

Maria VIDAGAÑ. Universidad de Zaragoza (España). mavimur@unizar.es

Verónica SORIA. Universitat Politècnica de València (España).

verosoma@gmail.com

Neus LOZANO. Universitat Jaume I (España).neus.lozano@uji.es

Resumen: El siguiente artículo pretende identificar posibles espacios comunes entre arte y educación partiendo de la propuesta artística Mapa dels Desitjos ${ }^{l}$ del colectivo Cràter $^{2}$. Esta propuesta se llevó a cabo en el contexto de la XVII Mostra d'Art Públic / Universitat Pública de la Universitat de València. Mapa dels Desitjos surgió de la idea de preguntar a la comunidad universitaria cómo organizarían su vida si no tuviesen en cuenta la cuestión económica y de hacer públicas sus respuestas. A partir de esta experiencia, analizamos y reflexionamos en torno a cuatro preguntas que estructuran el análisis: ¿Qué paralelismos existen entre los roles de artista/ público y maestro/estudiante? ¿Cómo se utiliza el espacio? ¿Cómo se comporta el factor tiempo? ¿Cuál es el conocimiento que se genera? Siguiendo las relaciones entre estos elementos, presentes tanto en el arte como en la educación, abordamos algunas reflexiones sobre conceptos educativos en permanente desarrollo, tales como educación expandida (Díaz et al., 2012), aprendizajes colaterales (González, del Rincón, Bonilla y Sáez, 2012) y currículum oculto (Freire, 1975).

Palabras Clave: Educación Artística, Investigación Artística, Práctica artística, Educación Superior, Entornos de aprendizaje.

\footnotetext{
1. http://mapadelsdesitjos.com/

2. http://lescrater.com
} 
VIDAGAÑ, SORIA \& LOZANO / Arte y Educación: Espacios comunes. Reflexiones en torno a la práctica artística Mapa dels desitjos

\begin{abstract}
The following paper aims to identify possible common spaces between art and education, departing from the art project Mapa dels Desitjos (Map of Wishes), by the collective Cràter. The project took place in the framework of the 17 Show of Public Art / Public University at University of Valencia. Mapa dels desitjos emerged from the idea of asking members of the university community about how they would organize their lives if they did not have to consider any economic constraints, and then making their answers public. Based on this experience, we analyze and reflect on four questions that structure our analysis: What paralels are there between the roles of artist/audience and teacher/student? How is the space used? What role does time play? What is the knowledge being generated? Through the relationships between these elements, present both in art as well as in education, we engage in some reflections on educational concepts that are in continuous development, such as expanded education (Díaz et al., 2012), collateral learning (González, del Rincón, Bonilla \& Sáez, 2012) and hidden curriculum (Freire, 1975).
\end{abstract}

Keywords: Artistic Education, Artistic Research, Artistic Practice, Higher Education, Learning Environments.

\title{
Introducción
}

La poesía nos libera de un mundo lleno de cosas útiles e inmediatas.

Nuno Júdice

La práctica artística desvela y sugiere, en algunas ocasiones, matices y luces que en otros contextos serían difíciles de apreciar. En este artículo, pretendemos iniciar una reflexión para establecer algunos de los lugares comunes existentes entre arte y educación a partir del proyecto Mapa dels desitjos. Consideramos esta reflexión posterior a la realización de la práctica artística como una oportunidad para analizar los nuevos saberes que nos ha aportado. Al mismo tiempo, esto constituye un acto imprescindible para construirnos como profesionales reflexivos (Schön, 1992) tanto a nivel artístico como docente.

En primer lugar, presentaremos el colectivo Cràter (Creación artística, Teoría, Educación y Recursos) con el fin de contextualizar el origen de la propuesta, y cómo nuestro funcionamiento trata de reflejar las colaboraciones posibles entre el profesorado. En segundo lugar, explicaremos Mapa dels desitjos y cómo se generan intercambios entre los integrantes de la comunidad universitaria así como cuáles son sus deseos comunes. Y en tercer lugar, presentaremos algunas de las reflexiones que surgen a partir de la experiencia a modo de preguntas, cuya respuesta nos permitirá expandir y enriquecer nuestras ideas sobre la generación y la adquisición de conocimiento en el proceso artístico y educativo. 


\section{Colectivo Cràter}

Cràter es un colectivo dedicado a la investigación y a la acción artística que toma como campo de estudio la práctica artística y la educación. Éste nace de la necesidad de compartir algunas inquietudes y experiencias comunes: el hecho de ser mujeres, estudiantes, licenciadas, masters, doctoras, docentes universitarias, investigadoras, trabajadoras precarias dentro y fuera de la academia, madres trabajadoras, y amigas. Es por esto que nos veíamos con la necesidad de generar una zona confortable, que nos sirviera como espacio de pensamiento y reflexión, dónde seguir autoformándonos en nuestro ámbito profesional como artistas-enseñantes.

Nuestro colectivo se ha encaminado a elaborar y compartir un discurso común y consensuado. En primer lugar, planteamos cuestiones desde nuestras vivencias, y esto nos sirve como primer paso para generar un proyecto cuya puesta en práctica utilizamos como material discursivo. Por tanto, la experiencia que se deriva de esta acción configura nuestra práctica artística y docente. La figura del cráter nos sirve como metáfora, en tanto que ésta, por su orografía, funciona como una boca. Que a su vez funciona como abertura de muchos otros. El cráter es el resultado de la explosión de todos ellos. Funciona como un altavoz que aúna y amplifica todas las tensiones y corrientes que se generan en el interior y que desembocan en la superficie.

Durante los dos años de existencia, hemos concentrado nuestros esfuerzos en la elaboración de proyectos como Mapa dels desitjos (Mostra d'Art Públic per a Joves Creadors "Art Públic / Universitat Pública"), Derives en torn a la ciutat. La natura com a resistència (Parlem d'Art, 34 Gallery y Universitat de València), 1,2,3 Referents (MiniIntramurs, Festival per l'art a València), Taller de Usos de herramientas artísticas para activar y potenciar la participación en el aula (Universidad de Verano de Teruel), etc.

\section{Mapa dels desitjos}

Mapa del desitjos es una intervención que realizamos paralelamente en el espacio público y en el entorno web. Se presentó durante la Mostra d'Art Públic per a Joves Creadors Art Públic / Universitat Pública dentro del marco del Festival de Benvinguda de la Universitat de València, en el Campus de Tarongers del 26 de septiembre al 3 de octubre de 2014. Nuestra propuesta consistió en realizar entrevistas a la comunidad universitaria, preguntándoles cómo organizarían su vida si tuviesen resuelta la cuestión económica. Las respuestas a esta pregunta fueron posteriormente compartidas en el Campus de Tarongers y a su vez en el entorno web Mapa dels desitjos ${ }^{3}$. A continuación vamos a precisar cuál fue el planteamiento inicial del proyecto, los aspectos técnicos y el desarrollo de cada una de las etapas del proyecto. 
VIDAGAÑ, SORIA \& LOZANO / Arte y Educación: Espacios comunes. Reflexiones en torno a la práctica artística Mapa dels desitjos

Planteamiento inicial del proyecto.

El planteamiento inicial de esta propuesta era el de articular un diálogo entre los integrantes de la comunidad educativa (en este caso la Universitat de València) e incorporarlo en el espacio compartido, para que pudiera darse una continuación de este intercambio en el tiempo. Al principio, pensamos en diferentes maneras de hacer visible este diálogo, como colocar escritas las reflexiones de los participantes, para que pudiesen generarse preguntas entre los transeúntes. Esto formaba parte de un esfuerzo por hacer visible los aprendizajes invisibles que tienen lugar en la institución educativa, en la transmisión de normas, valores y creencias que se adquieren. Esto es, evidenciar partes del currículum oculto (Giroux, 1983) y de los aprendizajes colaterales (González, del Rincón, Bonilla \& Sáez, 2012). El desafío estaba en presentarlo de manera plástica, que tuviera una conexión con las artes visuales.

Por ello, pensamos en cómo podríamos exhibir directamente los diálogos producidos. Para hacer esto, tuvimos la idea de crear un espacio virtual superpuesto al espacio compartido, en que pudieran alojarse las entrevistas. De esta manera, hallamos una forma de, literalmente, dar voz a las personas que generalmente no pueden ser escuchadas en este entorno y además dar cabida a este intercambio en el espacio público. Este intercambio versaría sobre procesos personales de aprendizaje. De nuevo, se trata de hacer lo invisible visible. Por otro lado, queríamos estudiar qué relación hay entre estos procesos y el espacio común.

Como se puede leer en la página $\mathrm{web}^{4}$ del proyecto, nuestras ideas se desplegaban en torno a una pregunta principal, que lanzamos a los entrevistados para iniciar una conversación en torno a las metas y al espacio compartido:

"¿Qué pasaría si viviéramos en una sociedad donde en lugar de guiarnos por la eficacia y la eficiencia nos guiáramos por los deseos? Esta pregunta, lejos de aspirar a ser una reivindicación, nos sitúa al comienzo de un juego que queremos proponer.

Con la finalidad de hacer partícipes de nuestro proyecto a los miembros de la comunidad universitaria, (estudiantes y docentes), proponemos realizar una serie de entrevistas para conocer y poner en común los deseos de esta comunidad.

Estamos acostumbrados a responder encuestas que tienen como fin ser útiles para algún propósito, predecir los resultados de unas elecciones, hacer un estudio de mercado, conocer los hábitos de consumo, etc.

No obstante, en esta propuesta, más próxima a la ficción literaria que a cualquier otra cosa, queremos indagar en los deseos de la comunidad universitaria y hacer una puesta en común».

4. http://mapadelsdesitjos.com/proyecto.html 
Por todas estas razones, queríamos generar en primer lugar una reflexión que abordara las metas de las personas que integran la comunidad educativa, para ver la importancia de los objetivos a medio y largo plazo, así como qué papel desempeñan estos en el aprendizaje y la generación de conocimiento. Pero al mismo tiempo, la cuestión de cómo estas actividades se desarrollan en el espacio destacaba igualmente por su importancia, en tanto que el espacio puede proporcionar lugares de intercambio de ideas, o puede aislar a las personas.

Aspectos técnicos y desarrollo de las etapas del proyecto

La realización de Mapa dels desitjos se llevó a cabo en dos etapas. En una primera etapa realizamos entrevistas a la comunidad universitaria del Campus de Tarongers de la Universitat de València, esto es: a estudiantes y profesorado de las facultades de Magisterio, Economía, Ciencias Sociales y Derecho. Les preguntamos cómo plantearían su vida si tuviesen resuelta la cuestión económica. Las respuestas de los estudiantes y del profesorado fueron grabadas en audio y ubicadas en un entorno web expresamente diseñado para la propuesta artística. Hicimos un total de 45 entrevistas. Las entrevistas se hicieron a integrantes de la comunidad universitaria en un sentido amplio. No obstante, a todos se les realizó la misma pregunta. Precisamente es en las diferentes maneras, desde cada posición individual, que se contesta esta pregunta, que la escucha es más interesante y variada. Se puede acceder al archivo de audios en la web de Mapa dels desitjos ${ }^{5}$

De igual manera, preguntamos a cada entrevistado su lugar favorito del campus y los motivos de su elección. Esto nos sirvió para distinguir qué factores son importantes (y generalmente comunes) para que estas personas prefieran estos lugares. Por ejemplo, muchos de los entrevistados manifestaron la necesidad de lugares donde poder encontrarse con otras personas, aspecto que la arquitectura de las facultades y la estructura de los edificios no siempre propicia. En el otro extremo, muchos manifestaron la falta de lugares donde estar tranquilo, sin sentirse observado, lo que también se da de manera inusual en el campus.

La segunda etapa consistió en adjudicar un código QR a cada una de las entrevistas y en hacer unos carteles con cada uno de los códigos QR. Cada cartel con su QR fue colocado en el lugar preferido de cada una de las personas entrevistadas en el Campus de Tarongers de la Universitat de València. Al construir un mapeado de estos lugares escogidos, se generaba un itinerario, que reflejamos en un mapa que se repartió a los transeúntes con el objetivo de indicar claramente dónde estaban ubicadas cada una de las entrevistas. De esta forma, las entrevistas permanecían ancladas en el espacio público del campus durante la semana de bienvenida de la

5. http://www.mapadelsdesitjos.com/index_esp.html 
VIDAGAÑ, SORIA \& LOZANO / Arte y Educación: Espacios comunes. Reflexiones en torno a la práctica artística Mapa dels desitjos

UV, y podían ser escuchadas por cualquier persona que tuviese un teléfono con lector de códigos QR.

Además, en lo que respecta a la generación de conocimiento, distinguimos una tercera fase, que es la que sucede después de que la obra tiene lugar. Durante la semana de bienvenida, los transeúntes tuvieron la oportunidad de pasear por el campus e interactuar con la obra. De manera permanente, en la página web se halla un archivo y un mapa de sonidos, donde se puede acceder a las entrevistas en su totalidad, al pinchar en el marcador que designa su lugar favorito. Es una vez que las personas han podido visitar la obra en el espacio físico y en la web, en la reflexión y el posible diálogo que se genera con el público participante, acerca de los objetivos y del papel del espacio en el aprendizaje, que se completa la obra.

Lugares comunes entre Arte y Educación.

Mapa dels Desitjos nos hace reflexionar sobre los límites establecidos entre arte y educación. ¿Dónde se ubican los límites del arte y dónde los de la educación en algunas de las prácticas artísticas actuales?

Entendemos la educación desde una perspectiva expandida (Díaz et al., 2012), que consiste en integrar otros espacios de aprendizaje para nutrir los contenidos de la educación formal, con saberes, reflexiones y perspectivas no formales, que se añaden a los currículums formativos como estrategias de aprendizaje. Así, podemos afirmar que en Mapa dels desitjos la cuestión educativa está totalmente implícita. En el catálogo Un saber realmente útil (What, How \& for Whom et al., 2014), se recoge:

El concepto de saber realmente útil surgió a comienzos del siglo XIX. cuando los obreros tomaron conciencia de la necesidad de la autoformación. En las décadas de 1820 y 1830, las organizaciones obreras de Reino Unido introdujeron esta frase para describir el corpus de conocimientos que abarcaba diversas disciplinas poco prácticas como la política, la economía y la filosofía, caracterizadas como opuestas a los saberes realmente útiles, proclamados como tales por los empresarios, que habían empezado a invertir cada vez más en el desarrollo de sus negocios mediante la financiación de programas formativos destinados a los obreros en competencias aplicables y disciplinas como la ingeniería, la física, la química o las matemáticas.

En este sentido, Mapa dels desitjos, al hacer a los integrantes de la comunidad universitaria plantearse sus objetivos individuales y presentarlas en relación al conjunto de esta comunidad, trata de devolver al contexto de la educación universitaria enseñanzas que plantean el papel del individuo dentro del contexto social, opuestas a las enseñanzas regladas, efectivas para el mercado laboral, que tienen un papel central en el sistema educativo. En un momento en el que la filosofía está quedando relegada en el sistema educativo y existe presión para reducir la educación artística al diseño y a otras disciplinas aplicadas, estos planteamientos se hallarían de otro modo ausentes en la universidad. 
Pero además, por los paralelismos que se producen entre el funcionamiento de esta obra y sus interrelaciones con el proceso de aprendizaje, Mapa dels Desitjos nos sirve como punto inicial para la discusión en torno a la idea de prácticas artísticas en las que la cuestión educativa se halla totalmente implícita y en las que arte y educación habitan lugares comunes. Estos paralelismos que se producen entre los roles de artista/público y maestro/estudiante son los siguientes:

En el arte contemporáneo se dan lugar una serie de puntos de inflexión durante las dos últimas décadas del siglo XX y comienzos del siglo XXI, que conllevan unas prácticas a menudo descritas como arte público de nueva generación. Algunas de estas prácticas se recogieron en España en el volumen Modos de hacer (Blanco, 2001). En este tipo de obras, la idea de público sufre una transformación, son los espectadores los que participan en la generación de los contenidos de la obras. De igual manera, en las prácticas educativas, el rol de los estudiantes cambia, y son ellos los que se responsabilizan de la generación de conocimiento (Rogoff, 2007).

En este sentido, estas personas no son solamente receptores, sino productores del discurso que se genera en la obra. Lucy R. Lippard define el arte público como "cualquier tipo de obra de libre acceso que se preocupa, desafía, implica, y tiene en cuenta la opinión del público para quien o con quien ha sido realizada, respetando a la comunidad y al medio" (Lippard, 2001). Además, Nicolas Bourriaud (2008), en Estética Relacional, explica una serie de prácticas artísticas en que la obra se configura a partir del vínculo que se crea con el público. De manera similar, sin el público, Mapa dels desitjos no existiría, puesto que son las propias voces de las personas que desarrollan su actividad en el espacio en que se emplaza la obra y la posterior escucha de estas grabaciones las que componen la acción artística.

Estos principios se hallan inscritos en la obra de artistas como Antoni Abad, Democracia, Jens Haaning, Roger Bernat o Nicolás Paris. Por ejemplo, en las prácticas de Antoni Abad con distintos colectivos 6 , (colectivo de taxistas en Mexico, trabajadores del sexo en Madrid, jóvenes gitanos en Lleida) en el que el supuesto público es parte activa en la creación de los contenidos de la propuesta artística. Y por consiguiente experimenta un aprendizaje significativo. El propio Antoni Abad manifiesta que el público en sus propuestas artísticas pasa a convertirse en usuario (Abad, 2010). Por poner solo un ejemplo, el colectivo de taxistas en México estaba totalmente estigmatizado por los medios de comunicación y, gracias a este proyecto, tuvieron la oportunidad de crear su propio canal de comunicación, pudiendo ofrecer su visión de su realidad. Igualmente, en las propuestas de Roger Bernat, el público tiene un papel totalmente activo. Concretamente en la práctica artística Domini

6.http://megafone.net/site/index 
VIDAGAÑ, SORIA \& LOZANO / Arte y Educación: Espacios comunes. Reflexiones en torno a la práctica artística Mapa dels desitjos

Públic ${ }^{7}$, los participantes, a través de las respuestas que da a diferentes cuestiones, se redistribuye en el espacio, y experimenta a través de su cuerpo y de las relaciones cambiantes que se generan con el resto de integrantes. De esta forma el público obtiene experiencias significativas que consideramos que están totalmente ligadas a la idea de aprendizaje.

En cuanto al uso y significación del espacio compartido, el espacio se revela como un parámetro artístico de una marcada relevancia. Como demuestran el concepto de campo expandido en el contexto de las prácticas artísticas, de las cuales son ejemplo la instalación, y diversas manifestaciones del arte público, "la lógica espacial de la práctica posmodernista ya no se organiza alrededor de la definición de un medio dado sobre la base del material o de la percepción de éste, sino que se organiza a través del universo de términos que se consideran en oposición dentro de una situación cultural" (Krauss, 1979). En concreto, las prácticas que nos han servido de referencia para nuestro proyecto tienen en común que generan una reflexión sobre el espacio compartido con el fin de unir a la comunidad en una experiencia. En este sentido fueron los artistas relacionados con la Internacional Situacionista los que sirvieron de referencia para este trabajo. En concreto, las reflexiones en torno a la psicogeografía y el paseo como ejercicio de toma de conciencia del espacio.

De manera similar, el espacio en que tiene lugar el aprendizaje es determinante, pues si los estudiantes no sienten seguridad y pertenencia al sitio no es posible la participación libre y la generación de conocimiento. Por ello, nos planteamos preguntarle a las personas que generalmente utilizan el espacio del campus acerca de sus preferencias sobre este en tanto que espacio, y utilizarlo al mismo tiempo para emplazar sus propias reflexiones. Así el espacio es el referente, el objeto y la situación misma de la obra. Precisamente en un momento en que la arquitectura de las universidades modernas propicia la eficacia del uso del espacio, en detrimento de los encuentros casuales, el paseo y la actuación espontánea en el perímetro de sus edificios, nos parecía importante inquirir a los estudiantes y a los trabajadores de estos espacios cómo se sentían respecto a ellos, e invitar posteriormente a los miembros de la comunidad a caminar por este espacio, al tiempo que visitaban las reflexiones de otros y --posiblemente-- se hacían ellos mismos idénticas preguntas.

Otro paralelismo entre esta práctica artística y la práctica educativa es el factor tiempo. En todo aprendizaje se requieren tres fases básicas. La primera, en que se genera una motivación y una finalidad, y se revela como indispensable el deseo del estudiante de adquirir un conocimiento; la segunda, en que se realiza la interacción con el material y la generación de conocimiento; y la tercera, en que se realiza una reflexión sobre lo aprendido, a modo de autoevaluación. Estas tres fases se identifican con las descritas en Mapa dels desitjos. En la primera fase, en que

7. http://rogerbernat.info/en-gira/domini-public/ 
preguntamos a los estudiantes, les hacemos (idealmente) manifestar una inquietud respecto a su situación y al espacio en que se encuentran. En la segunda fase, en que exponemos la obra, los miembros de la comunidad educativa interaccionan con el espacio en que se hallan distribuidas las manifestaciones de los entrevistados, en forma de grabaciones y a las que pueden acceder mediante sus dispositivos móviles. En la tercera fase, es en la que nos encontramos ahora mismo, extrayendo conclusiones y conocimiento a partir de la experiencia vivida.

Es siguiendo esta tercera fase que llegamos al debate acerca del conocimiento generado, sobre el currículum y el currículum oculto, y los aprendizajes colaterales. En la obra, se diferencia entre las metas que los entrevistados se ponen a título personal, y los aprendizajes que acaban sucediendo en sus vidas, siempre con el condicionante de su situación económica. Al analizar esta pieza, podemos darnos cuenta de la importancia de los objetivos, ya que estimulan el deseo de conocimiento en el estudiante. Sin embargo, a menudo desestimamos aquellos aprendizajes que no tienen una finalidad práctica para el desempeño de las competencias que forman un profesional, y sin embargo son estos los que son de verdad importantes para el estudiante (González et al., 2012). En Mapa dels desitjos, hemos querido poner de relevancia una reflexión sobre este tipo de conocimientos, en relación con la idea antes mencionada de los saberes realmente útiles (What, How \& for Whom et al., 2014).

\section{Conclusiones}

La realización de Mapa dels Desitjos, entre otras cosas, nos ha permitido reflexionar en torno a los lugares comunes entre arte y educación. Concretamente, las cuestiones que nos hemos planteado son: ¿Qué paralelismos existen entre los roles de artista/ público y maestro/estudiante? ¿Cómo se utiliza el espacio? ¿Cómo se comporta el factor tiempo? ¿Cuál es el conocimiento que se genera?

La propuesta comenzó entrevistando al público, pero con una pregunta le hizo exponer una reflexión acerca de sus objetivos personales y su planteamiento de vida. Pero además, esta pregunta también nos sirvió para cuestionar cómo el aprendizaje se entroncaba con las metas personales de los estudiantes. Por ejemplo, en ocasiones, algunos estudiantes manifestaron estar estudiando para satisfacer sus necesidades materiales, aún sin saber si en el panorama económico del momento estos estudios les harían llegar a un puesto de trabajo. Sin embargo, en su mayoría, las personas contemplaron la posibilidad de dedicarse a fines altruistas, una vez tuvieran sus necesidades satisfechas. De manera similar, algunos de ellos se encontraban descontentos con el aprendizaje que se daba lugar en la universidad, pues no encontraban una identificación del conocimiento adquirido con sus necesidades futuras.

Posteriormente, en una segunda fase, en que se expuso la obra, hubo una puesta en común y un intercambio de reflexiones individuales. Esta puesta en común se 
VIDAGAÑ, SORIA \& LOZANO / Arte y Educación: Espacios comunes. Reflexiones en torno a la práctica artística Mapa dels desitjos

realizó a través de la ubicación de las entrevistas en el espacio público del campus. De esta manera, cualquier transeúnte pudo durante una semana pasear por este espacio y escuchar las entrevistas a través de su teléfono móvil. Para nosotras, esto era muy importante porque el público receptor/productor de discurso podía adquirir conciencia de cómo sus necesidades, aun únicas, tienen muchos aspectos en común con otros integrantes de la comunidad y responden a anhelos en común e insatisfacciones que se generan de manera generalizada en nuestra sociedad.

En cuanto a la tercera fase, es ahora, una vez terminada la práctica cuando podemos realizar una reflexión sobre ella y evaluar la experiencia vivida, y con ello, darnos cuenta de cómo estos objetivos personales tienen una importancia en el proceso de aprendizaje. Estos objetivos que sirvieron como motor del aprendizaje, sirven para evaluar el conocimiento adquirido, y para dar cuenta de esos otros aprendizajes que no estaban planificados, pero que se han producido por el camino y han sido igualmente importantes.

Es por estas tres fases, en que los límites entre los roles del artista-emisor y del público-receptor se diluyen, para colaborar en la producción de una experiencia generadora de conocimiento en común, que Mapa dels desitjos puede servir como un ejemplo de lugares en común que las prácticas artísticas pueden tener con las nuevas prácticas educativas, en las que los roles tradicionales del maestro y el alumno también se difuminan y se genera un conocimiento consensuado, de mayor relevancia para los estudiantes que un aprendizaje unidireccional planificado exclusivamente por el profesor.

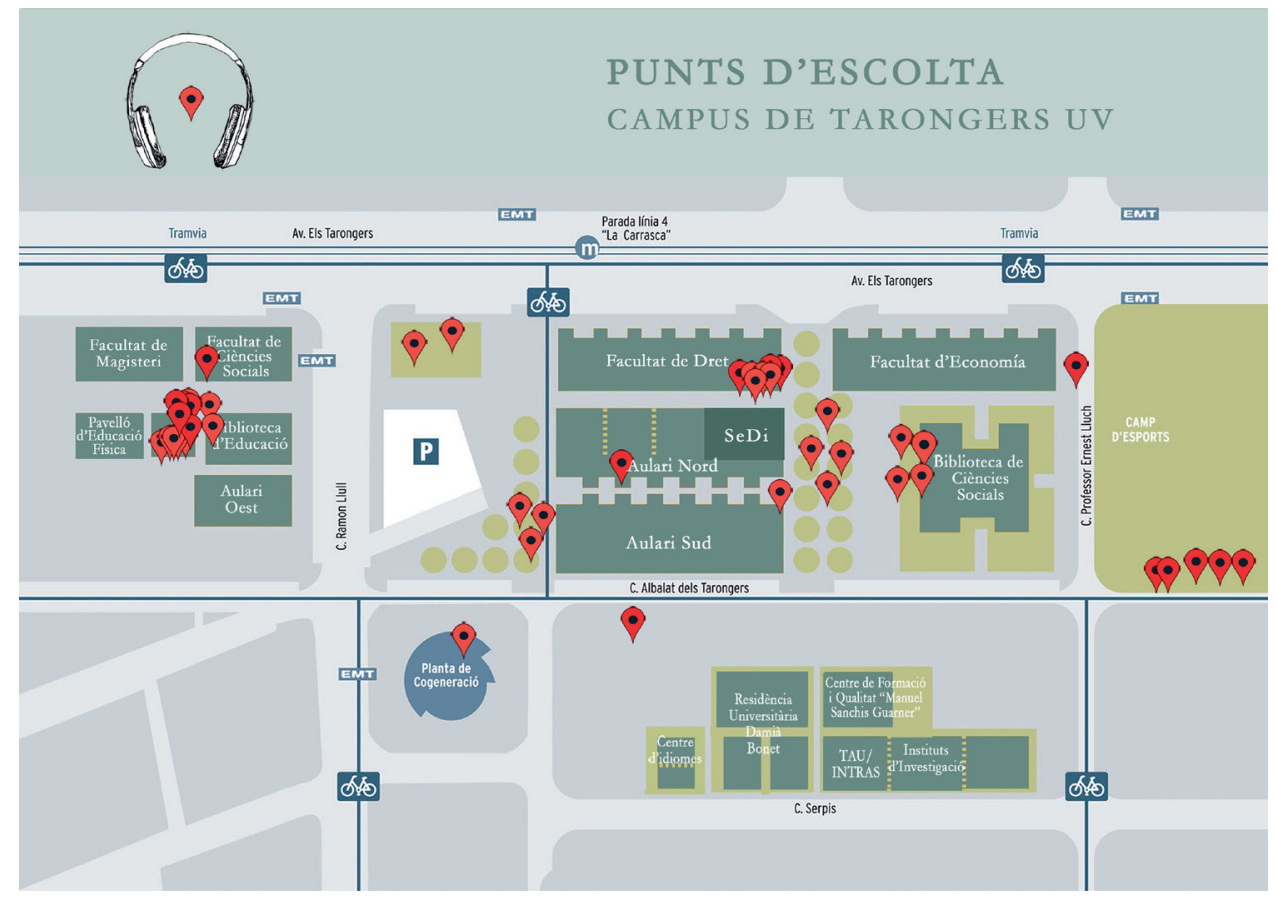

Figura 1. Puntos de escucha 
VIDAGAÑ, SORIA \& LOZANO / Arte y Educación: Espacios comunes. Reflexiones en torno a la práctica artística Mapa dels desitjos

\section{Referencias}

Abad, A. (2010). Cuando los públicos son los protagonistas. En Silvestre, R. (coord.), Arte contemporáneo y público. ¿Una relación imposible? (pp. 101-110). Valencia: Universitat de València.

Blanco, P., Carrillo, J., Claramonte, J., y Expósito, M.(Eds.). (2001). Modos de hacer: Arte crítico, esfera pública y acción directa. Salamanca: Universidad de Salamanca.

Bourriaud, N. (2008). Estética relacional. Buenos Aires: Adriana Hidalgo.

Díaz, R., Freire, J., Lamb, B., Martín, J., Lafuente, A., Wesch, M., De la Torre, A. (2012). Educación expandida: Festival Internacional Zemos98 - 11 edición. Sevilla: Zemos98.

Freire, P. (1975). Pedagogía del oprimido. Madrid: Siglo XXI.

Giroux, H. y Penna, A. (1983). Social Education in the Classroom: The Dynamics of the Hidden Curriculum. En Giroux, H. y Purpel, D. (Eds.) The Hidden Curriculum and Moral Education. Berkeley, California: McCutchan Publishing Corporation (pp. 100-121).

González, J.L., del Rincón, B., Bonilla, A.A., y Sáez, J.M. (2012). Aprendizajes colaterales: límites y retos del aprendizaje por competencias. En ENSAYOS, Revista de la Facultad de Educación de Albacete, núm. 27. Recuperado de http:// www.revista.uclm.es/index.php/ensayos.

Haghighian, N.S. (2011). Deshacer lo investigado. En Verwoert, J., Haghighian, N.S., Echevarria, G., García, D., Lesage, D., y Brown, T., En torno a la investigación artística. Pensaryenseñararte: entrelaprácticay la especulaciónteórica. Barcelona: Museu d'Art Contemporani de Barcelona y Universitat Autònoma de Barcelona.

Lippard, L. R. (2001). Mirando alrededor: dónde estamos y dónde podríamos estar. En Blanco, P., Carrillo, J., Claramonte, J., y Expósito, M. (Eds.), Modos de hacer. Arte crítico, esfera pública y acción directa. Salamanca: Universidad de Salamanca.

Pérez, J. L. (com.). (2015). Art Públic Universtitat Pública. XVII Mostra d'Art Públic per a Joves Creadors, catálogo de exposición. Valencia: Universitat de València.

Rogoff, I. (2007). La academia como potencialidad. Zehar: revista de Arteleku-ko aldizkaria, núm. 60/61 (pp. 10-15).

Schön, D. (1992). La formación de profesionales reflexivos: hacia un nuevo diseño de la enseñanza y el aprendizaje en las profesiones. Barcelona: Paidos Ibérica.

What, How \& for Whom, Garcés, M., Raqs Media Collective, Camnitzer, L., Paglen, T., Appelbaum, J., Moten F. y Harney S., y Tamás, G.M. (2014). Un saber realmente útil. Madrid: Museo Nacional Centro de Arte Reina Sofía. 